At all events I had some difficulty in expanding the blades of the instrument, although $I$ had thrown into the organ the quan. tity of water I usually inject-viz., about four ounces. However, I caught the stone, and while screwing home the blades, I perceived blood flowing somewhat freely from the orifice of the urethra. This determined me to desist, and I withdrew the instrument. For some time bloody urine continued to flow from the canal; but on the second day I was summoned by his medical attendant, in consequence of an attack of retention of urine, and I drew off about a pint and a half of urine, deeply coloured with blood. Retention again followed, and T removed nearly the same quantity, and of the same character of urine, on the fourth day. The presence of the distended bladder did not appear to cause him much inconvenience, nor its evacuation much relief. Urine, more or less bloody, continued to escape from the urethra without the effort of micturition. He had no local pain, and bore pressure, both over the bladder and on the perinæum, without complaint. He was ordered gallic acid in full doses. Sir Benjamin Brodie saw him at this stage, and did not augur unfavourably of my patient's case. He recommended the employment of Ruspini's styptic, and suggested the repetition of the operation as early as permissible. His increasing weakness was aggravated by the excessive action of a moderate dose of castor oil, and although the hæmorrhage was reduced in quantity, his vital powers were now only sustained by the frequent employment of stimulants. Bladders of ice were applied to the epigastrium and to the perinæum without effect. He became comatose, and died on the fifteenth day from the second operation.

The post-mortem examination exhibited a contracted bladder, thickened, with its inner surface coated with coagulum; a cal. culus of about the size and form of a moderate-sized walnut, broken into three parts-its composition, oxalate of lime coated with phosphatic salts, and two lesser calculi entire; ureters dilated; kidneys diseased.

In my work on "Operative Surgery," I have referred to two or three similar cases to that of $\mathrm{Mr}$. - but they are rare The fatal issue in this instance is not to be cited as injurious to the good name of lithotrity. Had the old operation by the knife been substituted, the issue had, in all probability, been the same. The presence of albumen in the urine, when coupled with stone in the bladder, is not conclusive evidence of diseased kidney, and with symptoms of an urgent character we are compelled to make the effort to obtain relief. The operation of lithotrity, when carefully performed, creates little more pain than that of sounding for stone, and generally, in a healthy bladder, leaves as little irritation behind. The proportion of persons who suffer from complications of renal disease, coupled with calculi, is small. I do not think such cases can be safely treated by the lithotrite. Neither is the cutting operation a security against a fatal termination.

Believing as I do that the operation by means of the lithotrite is applicable to the large majority of calculous affections of the bladder - that, if well executed, it is safer as regards the life of the patient, quite as certain as regards entire recovery, and less exhausting to the system, I recommend its practice for your consideration. And as I may not have the opportunity of addressing you again for some time, I purpose concluding these remarks with some general rules which may prove useful in your future career.

I advise your rejection of cases for lithotrity presenting the following characters:-

1. Manifest disease of the kidney.

2. The urethra so contracted as not to admit with facility a lithotrite of ample size.

3. The bladder so intolerant as to be incapable of retaining its urinous contents for three or four hours; and, on the other hand, a bladder of low nervous susceptibility.

4. Much enlargement of the prostate gland.

The quantity of water injected should not exceed four or five ounces. In many subjects, the employment of chloroform excites the bladder to contract, and the injection has to be repeated. The lithotrite, from its full size and angular form, should be introduced with more caution than is usually required on the introduction of a catheter. No attempt should be made to open the instrument in the bladder until it has been pushed thoroughly home into the organ. In the act of separating the blades, do not withdraw the upper, without at the same moment pressing the lower blade downwards towards the bottom of the bladder. If this rule be not strictly observed, the upper blade will be painfully pressed against the neck of the bladder, from which hæmorrhage may follow. The stone is to be brought into the lithotrite by pressing the lower blade suddenly, and by a slight jerk or twist of the hand, against the base or bottom of the bladder. There is neither necessity nor advantage in direct ing the instrument to the right or to the left. It should retain the mesial line throughout the entire operation. When the stone is caught, the blades should be screwed "home," lest small accumulations become large, and render the withdrawal of the instrument through the urethra difficult. At the first operation, do as little as possible. It may be deemed an ex. perimental occasion, and it will be sufficient to break the stone once across. On all future occasions, the number of applications of the serew may be determined by the tolerance of the patient. The stone may be broken six, eight, or more times. The intervals between each operation will vary accord. ing to the condition of the bladder, and the quantity of detritus expelled. If the quantity be considerable, and the bladder quiet, the operation may be repeated in four or five days. The average interval is longer than this. Few cases pass through their course of treatment without giving evidence of irritability of the mucous membrane of the bladder, manifested by a discharge of tenacious mucus adhering to the bottom of the vessel. Unless in its aggravated form, it is not a serious symptom. It may be treated with diosma, or uva ursi, nitrie acid, Dover's powder, \&c.; but the best remedy is the litho. trite. I have repeatedly seen this symptom subside on the repetition of the operation. The constitutional treatment is chiefly dietetic. Diluents should be ordered largely; and the moderate use of wine is unobjectionable. I have never seen any advantage obtained by an abstinent diet, nor any evil arise from an ordinary and habitual one. It is surprising how large a fragment may travel along a healthy urethra. They are arrested, however, most frequently at the glans, and if a frag. ment cannot be extracted by a pair of fine forceps in this situation, the urethra should be divided. When fixed low down in the urethra, they should be pushed back into the bladder. This may be effected by a large catheter (No. 12), cut off straights at the point, the extremity of the instrument being supplied by a movable knob, which is withdrawn when the catheter touches the stone. The open extremity of the instrument encircles the stone, which is forced backwards without injury to the mucous membrane. A small abscess in the perinæum may occasionally follow the violent employment of the lithotrite, or forceps, \&c. It presents itself under the form of a small rounded tumour. It seldom requires active treatment, and, as a general rule, may be "let alone."

\section{ON A CASE OF ANGINA PECTORIS. WITH PEMARKS.}

BY J. MOORHEAD, A.M., M.D., Weymouth.

ON the 24th of January, 1859, I was requested to visit Mr. - - aged sixty-three, who, I was informed, suffered from some painful affection of the chest. On my entering his bedroom, he suddenly burst into tears, which, however, lasted only for a few minutes. Such manifestations of feeling, he said, were quite beyond his control, and had been easily induced ever since he had had two or three slight paralytic seizures several years ago. When the emotional excitement was allayed, he stated that when walking, and especially soon after meals, he was suddenly seized with a painful sensation in the præcordial region, which soon became aggravated to an intense degree. This feeling of anguish was accompanied by an aching pain shooting down both arms, and extending even to the tips of the fingers. It came on more particularly when he walked up a hill or against the wind. On the pain becoming so severe as to be no longer bearable, he stood still, and it almost immediately vanished. A similar pang recurred after a little further exercise, and was removed by a like proceeding. During the paroxysm, his breathing, he said, was maffected.

From these attacks he had suffered for nearly fifteen years; but his disease had latterly become so aggravated that the slightest physical exertion or transient emotion sufficed to induce a paroxysm. The bowels acted only under the influence of aperient pills. He was subject occasionally to flatulency and acidity of stomach, which invariably aggravated the angina, and sometimes, he thought, brought on a paroxysm.

His previous treatment chiefly consisted in the use of remedies ordinarily prescribed for dysuepsia, many of the medical men whom he consulted considering this his chief disorder, 
and the neuralgic pain of chest the effect. Having almost exhausted the catalogue of such remedies, he had been using for more than two months past Boudault's pepsine, from which, however, he had derived no benefit.

On examination of the chest, the percussion-note was fotnd resonant over both lungs, but there was a greater extent of præcordial dullness than usual. The respiratory murmur was natural. There were no cardiac bruits whatever, the only apparent deviation from health being faintness of the heart's normal sounds, with feebleness of its impulse; its beats were rhythmical, and the pulse at the wrist was 92 , weak. There was no dyspncea; no pain or tenderness in the epigastric or hypochondriac regions; no nausea or vomiting; tongue moist, and nearly clean.

During my visit he suffered severely from a paroxysm, which, however, was much alleviated by a dose of opium. Ordered an anodyne liniment, consisting of tincture of opium and chloroform, with compound camphor liniment, to be rubbed into the chest when the pain supervened; also an antacid mixture, containing dilute hydrocyanic acid in two-minim doses, to be taken three times a day.

Jan. 26th. - Feels better, and has not had paroxysms of angina so frequently. When they came on, he states he received much relief from the application of the liniment, and the use of morphia in small and repeated doses; sits daily in the drawing-room; has a slight cough, contracted since last visit. Stethoscope still reveals but negative signs of any cardiac derangement. Repeat mixture.

28th. - About eleven o'clock last night, the patient was suddenly seized with angina, together with dyspnoea, which gradually increased till the kreathing became very short and laboured. When I arrived (about one A.M.), the patient presented a haggard, anxious expression, with rolling eyes, indicative of his acute sufferings and intense dyspncea. Stethoscopic examination showed a constricted condition of the bronchial tubes, rhonchus and other sonorous râles being everywhere throughout the lungs distinctly audible. The suddenness of the attack, together with the absence of corresponding excitement in the vascular system (the pulse being weak and the extremities cold), led me to regard the dyspnoea as nervous, and accordingly $I$ administered the following draught:-Compound tincture of valerian, one drachm; aromatic spirit of ammonia, half a drachm; compound spirit of sulphuric ether, half a drachm; solution of muriate of morphia, fifteen minims; springwater, to an ounce and a half. In the course of a few minutes great relief was experienced; the rhonchus and other abnormal respiratory sounds could scarcely be detected, while the breathing became comparatively easy. Warm bottles were then applied to the feet, and the hands bathed in warm water. As the angina, however, persisted, the anodyne liniment was rubbed into the precordia, and solution of muriate of morphia, in twenty-minim doses, twice repeated. These induced sleep for a few hours. During this attack, the pain in the arms was entirely absent. -Twelve o'clock noon: Is free from acute pain, but complains of one of a dull, aching character in præcordia; has no dyspnœa, but occasionally expectorates a little mucus; bowels confined. Ordered, an ounce of castor oil; repeat mixture, substituting for the hydrocyanic acid "Hoffman's anodyne," in half-drachm doses, three times a day.

30th. - Looks better and feels cheerful; has had no return of paroxysm, nor even of dull pain of chest; bowels freely opened by the draught; no dyspncea, but a slight cough. Repeat last mixture.

Feb. 1st.-In drawing-room, and continues cheerful; still free from angina and dyspnoea; felt himself so much improved that yesterday he took a ride in a wheel-chair, which he much enjoyed; this he had not been able to do for three weeks previously; bowels open without the assistance of an aperient; appetite good; pulse feeble. Repeat mixture.

2nd.--Half-past six A.M. : Yesterday evening, dyspnœea came on, and continued during the night, but, as reported, not of so great urgency as in the former attack. Three small doses of an expectorant mixture were given at intervals, together with warm coffee; but these proving unavailing, a messenger was despatched for me. Unhappily, however, before my arrival, death took place. This event occurred so quietly, that I was assured the attendants were not aware of it till some minutes afterwards. Coupling this circumstance with the fact that the patient was able ten minutes before his dissolution to get up to the night-chair, it may, I think, be safely concluded that death took place by syncope.

A utopsy, thirty hours after death. (Assisted by Dr. Smith.) Cadaveric rigidity strongly marked; body corpulent, the subcutaneous layer of fat being about half an inch in thickness; sternal cartilages partially ossified; large amount of fat in mediastinum; about two ounces of straw. coloured fluid in pericardium; about two pints of serum in cavities of pleure; no pleuritic adhesions; lungs healthy, bat congested. Heart large (weighing seventeen ounces) and very fatty, its surface, especially on the right side, being so covered that its muscular tissue, which was thin and pale, was almost concealed from view; right cavities of heart contained a considerable quantity of liquid blood, the left much less; all the valves healthy and efficient; aorta normal in calibre and structure, with the exception of a very little calcareous deposit in the wall bounding one of the sinuses of Morgagni; the coronary arterits for an inch and a half from their origin were converted into rigid tubes, and presented beyond this, at short intervals along their course, as far as could be traced, specks of calcareous deposit. Stomach contained only a small quantity of liquid; its mucous membrane somewhat congested; otherwise healthy. Liver large and congested; gall bladder contained about an ounce of bile; spleen engorged with blood; kidneys healthy, but deeply congested; omenta loaded with fat. Head not examined.

While the foregoing case presents all the usual features of genuine angina pectoris, it has also some peculiar points which, 1 think, render it worthy of record. The duration of the disease is somewhat remarkable, extending, as before observed, over a period of nearly fifteen years. The most interesting point, however, is the urgent dyspnoea which occurred only on two occasions during that lengthened period. The first attack which I witnessed, on the 28th January, was so decidedly spasmodic that it may justly be assigned to the same cause as asthma, - namely, undue contraction of the muscular fibres of the bronchi. This is also shown by the almost immediate relief which ensued upon the administration of the antispasmodic draught. The spasmodic contraction of the bronchi, there is little donbt, was due to irritation conveyed along the motor fibres of the par vagum. What the source of that irritation was it is difficult positively to state, but the presumption is that it was the morbid condition of the heart. The implication of the motor filaments of the par vagum would seem to lend support to the theory that supposes that nerve, and not the sympathetic, to be the seat of angina pectoris. The absence of pain in the arms and the mild character of the angina during the attack of dyspnoea, show that the irritation of that nerve (par vagum) was then almost confined to its motor fibres.

Without, however, entering further into pathology, it may be stated that the morbid appearances exhibited in the above case were those most frequently found in connexion with this affection. The muscular tissue of the heart was thin and pale, and loaded with fat; while the coronary arteries were distinctly ossified. That these organic lesions excited the irritation of the par vagum which produced the angina can, I think, scarcely be doubted.

The other abnormal conditions may be explained by reference to the phenomena preceding death. The dyspnrea, which continued for several hours, brought on congestion of the Iungs, which led to accumulation of blood in the right chambers of the heart and in the entire venous system. Hence the congestion of all the abdominal viscera. The large quantity of serum in the pleural cavities can only be regarded as a post-mortem product, effusion being no doubt much augmented by the pulmonary congestion.

Although, as I have before stated, death took place by syncope, yet it is highly probable, from the great amendment in the symptoms, that that event would not have then occurred but for the dyspnœa. While syncope, then, was the immediate or proximate cause of death, dyspnoea, I believe, was its primary or remote cause.

July, 1859.

REPORT OF A CASE

OF

ELEPHANTIASIS (CRURIS) SUCCESSFULLY TREATED BY AMPUTATION ABOVE THE KNEE.

Br AUGUSTUS EVES, M.D., F.R.C.S., SENTOR SCRGEON TO THE CHELTENHAM GHNERAL HOSPITAL.

MARY J-, aged forty-six years, was admitted into the Cheltenham General Hospital July 27th, 1857. On examination, her left leg and foot were found greatly enlarged, the increase of size being most considerable at the foot, from which part it gradually lessened towards the knee. The enlargement 